\title{
THE NEED FOR A SELF-CONSISTENT MODEL OF THE ISM
}

\author{
JOEL N. BREGMAN \\ University of Michigan; E-mail: jbregman@umich.edu
}

\begin{abstract}
The interstellar medium of the Milky Way possesses a remarkable range of components, which have individual lifetimes considerably less than a Hubble time. Consequently, there has been a major effort in understanding the properties of these components, their heating and cooling mechanisms, and the process by which one component is transformed into another. We review several of the issues in an effort to highlight controversies that are under study presently. One of the central debates involves star formation, where there are two general and conflicting theoretical models. Another issue deals with the efforts to understand the fractional amounts of gas in the various phases, which brings together star formation with global gas dynamical reactions. This interaction is responsible for heating of the gas by supernovae, causing superbubbles, and subsequently, either galactic winds or galactic fountains. The relative amounts of material in the hot and cold phases not only depends upon the supernova rate, it also depends upon details of interstellar models, such as whether thermal conduction is an important process. We review the progress that people have made on self-consistent modeling of the ISM. The constraints on such models come from a wide variety of observations of molecular, neutral atomic, warm ionized, and X-ray gas. Both observations and theory are represented at this meeting and we highlight areas in which contributions are likely to resolve the existing controversies.
\end{abstract}

Keywords: ISM, models

\section{Introduction}

Occasionally a friend of mine will remind me that part of the study of the interstellar medium is much like the study of weather, which he says in a derogatory tone. However, I remind my friend that one of his favorite television channels is The Weather Channel, and he defends his fondness by telling me that knowing the weather is essential to planning his day. It doesn't really affect how he will teach his class that day, and he really watches because it is interesting. There are some similarities to our work in that it is simply an interesting field with wide-ranging implications. Of course, one of the big differences is that we do not have a television channel dedicated to the interstellar medium in galaxies and I wonder why that has not happened yet. On The Weather Channel, there are detailed forecasts based on numerical hydrodynamic models, and this seems to be one of the big attractions. So how far away are we from doing something similar: self-consistent models of the interstellar medium. This is the general topic of this conference, which has several parts. I will begin with a general census of the interstellar content of the Milky Way, with emphasis on some of the more controversial issues. Then, I will discuss the progress in the area of interstellar modeling, which has been more 
rapid then I would have predicted and is a testament to the inventiveness of the investigators.

\section{The Census of the Galactic ISM}

I will move from the components that I think we know the most about to those that we know least about, trying to highlight some of the outstanding issues involved.

\subsection{NeUtral ATOMIC GAS}

The component for which we have the most knowledge is probably HI, the cool atomic component of the ISM. Part of the reason for this is that $21 \mathrm{~cm}$ emission provides a linear measure of this gas component, so we have good masses, which is nearly a unique situation. For the other components, we measure either a surface brightness through an emission measure $\left(\mathrm{n}^{2} 1\right.$, where 1 is the lengthscale) or we measure an opaque surface ( $\mathrm{CO}$ emission). But for HI, not only is the optical depth low, but the velocity of the gas relates to the location in the disk of the Galaxy, so we obtain distance information as well.

Whereas the early picture of the HI was a "raisin-pudding" model with cool dense clouds imbedded in a more dilute intercloud, the current picture is that the cold, dense $\mathrm{HI}\left(10^{2} \mathrm{~K}, 30 \mathrm{~cm}^{-3}\right)$ is largely sheet-like (Kulkarni and Heiles, 1988). The HI between the clouds is warmer and of lower density $\left(300-3,000 \mathrm{~K} ; 1 \mathrm{~cm}^{-3}\right)$, but unlike the early picture, this does not occupy all of the volume. Some of the most remarkable images such as the the one shown in Figure 1, have been obtained with the Canadian Galactic Plane Survey, where images and references can be found at http://www.ras.ucalgary.ca/CGPS/gallery/.

The pressure is provided not only by the usual gas pressure but also by magnetic pressure and turbulent motion. The temperature of the gas is determined by the balance of photoelectric grain heating (a photon ejects an electron from a grain, which heats the gas) and radiative cooling from forbidden fine-structure lines (e.g., CI $157 \mu \mathrm{m}$ ) that are collisionally excited.

Despite the great strides that have been made, I have a few items on my "wish list' that I hope will be resolved in the future. The first has to do with the filling factor of the HI, where my best guess is $20-50 \%$, based upon the comparison of HI studies to models (Bregman, Kelson and Ashe, 1992). The other issue has to do with the High Velocity Clouds of HI, where three mechanisms have been proposed: galactic fountains; infall of gas from the LMC and SMC; and a population of gravitationally bound HI clouds that lie throughout the Local Group. There is good observational evidence that the first two mechanisms occur. For the infall from the LMC and SMC, there is little doubt that the Magellanic Stream has been torn away, and there is also good evidence that other HVC gas originated from the LMC based on its location and metallicity arguments (Wakker, Oosterloo and Putman, 


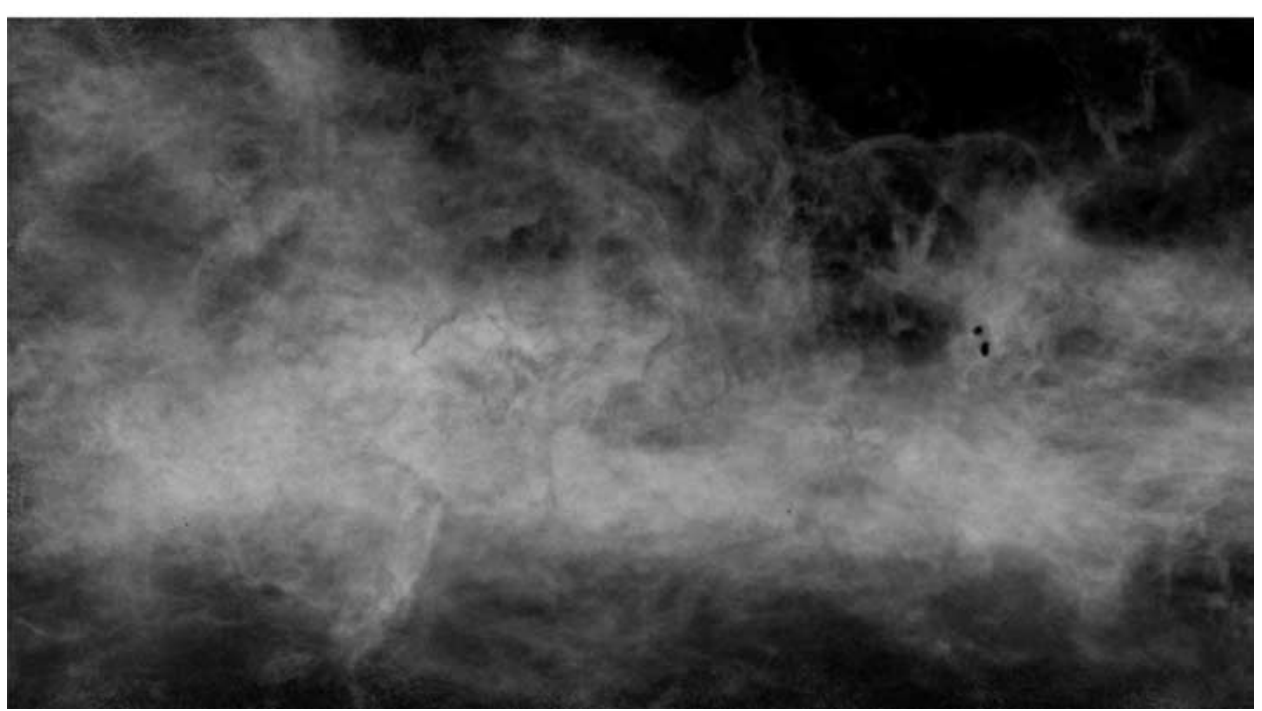

Figure 1. The HI total intensity map for the region of the Perseus arm in the Galactic plane (image size is $16^{\circ}$ by $9^{\circ}$ ) from the Canadian Galactic Plane Survey, taken with the DRAO array. This shows shells, sheets, and dense regions (the two small holes toward the right side are star forming regions filled with molecular gas and are bright IRAS regions).

2002; Wakker, this meeting). For the galactic fountain picture, we detect the hot X-ray emitting halos that are needed (both from the Milky Way and around other galaxies). The third models, Local Group clouds, predict an ensemble of about $10^{3}$ clouds of mass $5 \times 10^{7} \mathrm{M}_{\odot}$ with a typical distance of $500 \mathrm{kpc}$ (Blitz et al. 1999). Some problems with this suggestion is that such clouds are not seen in other galaxy groups, and optical emission line studies place the clouds at a distance closer than $100 \mathrm{kpc}$ and more likely 10-50 kpc (Weiner, Vogel and Williams, 2002; Tufte et al., 2002). However, the relative importance of these three mechanisms remains a hotly debated topic but one that can be resolved with further observations.

\subsection{WARM IONIZED GAS}

The warm ionized gas is a component that constitutes a significant amount of volume and mass in the ISM yet the study of this component has been led but just a few people. It is a component where we have an extremely good measure of the mass along sightlines because the pulsar dispersion measures are linearly proportional to the column of electrons (Taylor and Cordes, 1993). With their model for these dispersion measures, they can determine distances to targets given a dispersion measure (see their updated model and tool at http://pulsar.princeton.edu/ pulsar/converters.shtml). These dispersion measures imply an electron column density that is about $1 / 3$ of the HI column density, although with a scale height that is significantly higher, about $1.5 \mathrm{kpc}$ (Reynolds, 1989). The volume filling factor can be estimated from the comparison of emission and the dispersion measure and it is 


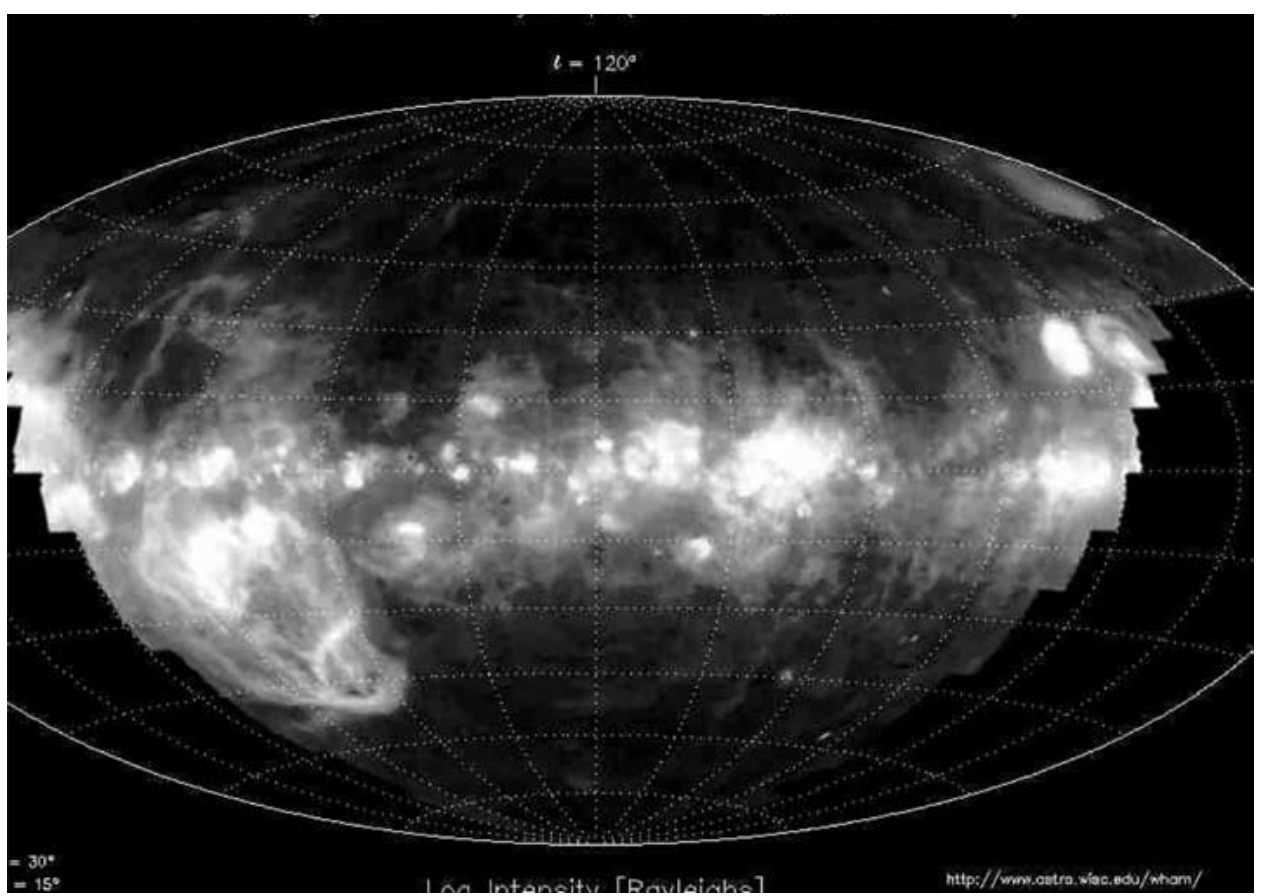

Figure 2. The $\mathrm{H} \alpha$ total intensity emission for part of the northern sky from the Wisconsin $\mathrm{H} \alpha \mathrm{Mapper}$ (WHAM). In addition to small bright regions that are the classical HII regions, one see diffuse emission from all locations as well as large structures that denote shells and chimney walls.

found to be about $20 \%$ (Reynolds, 1991), or possibly greater. The average electron density of this HI gas is $\mathrm{n}_{\mathrm{e}} \approx 0.08 \mathrm{~cm}^{-3}$, and if the temperature is about $6 \times 10^{3}$ $\mathrm{K}$, the pressure is $\mathrm{p} / \mathrm{k} \approx 10^{3} \mathrm{~K} \mathrm{~cm}^{-3}$, which is below the typical gas pressure and is significantly below the effective pressure associated with the colder gas (when including magnetic fields and turbulence, which leads to $\mathrm{p} / \mathrm{k} \approx 10^{4.3} \mathrm{~K} \mathrm{~cm}^{-3}$ ). Either these warm ionized regions are underpressure and are regions that are collapsing, or other forms of pressure (magnetic field, turbulence) plays a major role. A situation that could lead to underpressure warm ionized gas is the cooling of superbubbles from the hot phase $\left(10^{6} \mathrm{~K}\right)$, where they are in pressure equilibrium. Then, until these regions are compressed by their surroundings, they will have a pressure lower than the mean value for the ISM.

The WHAM survey (Reynolds et al., 1988) highlights lower density bubbles that are not the classical HII regions which one can see just from an examination of their survey data, shown in Figure 2 and updated on their home page http://www.astro.wisc.edu/wham/index.html. Some warm ionized regions are part of star-forming regions and photo-dissociation fronts (PDFs), which are among the most complex regions in the Galaxy, with the time-evolution of molecular, atomic, and ionized gas. Finally, we note that the heating of the warm ionized component is by photoionization while the cooling is largely from optical forbidden lines. 


\subsection{HOT IONIZED GAS}

Gas in the $2 \times 10^{4}-2 \times 10^{5} \mathrm{~K}$ range requires special conditions for gas to exist, whether it be in photoionization or collisional ionization equilibrium. The reason for this is that the cooling functions rise very quickly above $1 \times 10^{4} \mathrm{~K}$ and reaches a peak at about $2 \times 10^{5} \mathrm{~K}$, so the heating must fall in only a narrow range for thermal equilibrium. However, the collisional cooling function decreases from $2 \times 10^{5}$ $2 \times 10^{7} \mathrm{~K}$, so a single value of the heating function can lead to two equilibrium points at different temperatures. Also, the gas in this temperature regime is subject to a thermal instability that will cause the gas either to heat up or to cool down. Gas at about $2 \times 10^{5} \mathrm{~K}$ has a relatively short cooling time so this situation leads to a relative paucity of gas in the temperature range $10^{4}-10^{5.5} \mathrm{~K}$.

Continuous heating, such as by photoionization, rarely produces gas above $10^{4}$ $\mathrm{K}$ in the ISM, but shocks naturally produce hot gas in the $10^{5.5}-10^{7.5} \mathrm{~K}$ range. Supernovae, which dominate the energy input into the ISM, produce shocks with velocities of $100-1000 \mathrm{~km} / \mathrm{sec}$, and these shocks jump the gas temperature over the temperature regime of very rapid cooling and into the higher temperature regime.

The gas pressure of the shocked gas is greater than the surroundings, so it expands, as is seen in supernova remnants, superbubbles, and reheated low-density regions in the Galaxy. The all-sky mapping by ROSAT was essential for understanding the extent of the hot gas in the Milky Way. In addition to the Local hot bubble (100-200 pc), there are several other superbubbles of hot gas, possibly an extensive hot medium in the disk, along with a hot halo that is flattened (Snowden et al., 1998). The densities of this hot gas is typically $1-3 \times 10^{-3} \mathrm{~cm}^{-3}$ and the typical temperature is $10^{6} \mathrm{~K}$. The volume filling factor of the hot component in the plane of the Galaxy remains a topic of debate and we will hear much about it at this meeting. Recent calculations place the value at about $20 \%$, although the value is greater in the halo, where it may occupy most of the volume (de Avillez, 2000).

One of the new controversial topics relating to the hot component of Galactic gas is the extent of the halo and whether there is a hot group medium. Data has been accumulating for a few years, but the controversy really began last year when Chandra spectroscopic observations toward bright AGNs showed absorption lines of OVII and OVIII at zero redshift (Nicastro et al., 2002). Previously, OVI absorption was detected along many sight lines out of the Galaxy, which is detailed in a set of three comprehensive papers that describe the FUSE observations (Sembach et al., 2003; Wakker et al., 2003; Savage et al., 2003). Also, OVII was detected in emission in the X-ray band from a rocket flight (McCammon et al., 2002) and OVI was detected in emission in the UV band with FUSE (Shelton et al., 2000).

There are multiple ways of interpreting these data and one taken by Nicastro was to assume that the absorption by OVI, OVII, and OVIII were cospatial. This places several constraints on the nature of the ionization and they find that a pure collisional model will not fit the data, but they find that a low density $\left(10^{-6} \mathrm{~cm}^{-3}\right)$ system in photoionization equilibrium can reproduce the data. Since the column 
densities for these high ionization oxygen species are well-determined $\left(10^{16} \mathrm{~cm}^{-2}\right)$, this implies a size for the absorbing cloud of $4.9 /[\mathrm{O} / \mathrm{H}]_{\odot} \mathrm{Mpc}$, so for a metalliticy of 0.3 , it implies a lengthscale of $15 \mathrm{Mpc}$, much larger than the local group. Their solution that has the highest density and smallest column permitted lead to a lengthscale of $3 \mathrm{Mpc}$. This corresponds to about $5 \times 10^{13} \mathrm{M}_{\odot}$, more than an order of magnitude more mass than in all the known galaxies in the Local Group. This would have tremendous implications for the baryonic mass of the local universe if our Local Group is typical.

Alternatively, these three oxygen species may not be cospatial, as there is no compelling theoretical reason for such an arrangement. Instead, one might imagine that the OVII emission and absorption are cospatial, in which case, one obtains a minimum lengthscale of $50 \mathrm{kpc}$ (Sanders et al., 2002). Then, the OVI absorption is produced in a lower temperature region and there are few constraints on the lengthscale; it could easily lie within $100 \mathrm{kpc}$. For completeness, the OVI emission is believed to originate from the lower Galactic halo, within a few kpc of the midplane.

\subsection{THE MOLECULAR COMPONENT}

Finally, we come to the molecular component of the ISM, where the primary molecule by mass is $\mathrm{H}_{2}$. Unfortunately, it has no dipole moment, so it is a poor emitter and we are forced to find a proxy for it in emission. This substitute is usually $\mathrm{CO}$, where the rotational lines in the millimeter region are strong and reasonably accessible. The problem with this situation is that the $\mathrm{CO}$ lines are generally optically thick (such as the 1-0 line), so this is a poor tracer of the total mass. For giant molecular clouds, the line strength is probably a good measure of the mass, but as we discuss below, this is clearly not true for less massive molecular clouds, which are more common. The typical density of the giant molecular clouds is $10^{2} \mathrm{~cm}^{-3}$ and the temperature is around $10 \mathrm{~K}$. Magnetic fields are detected in these cloud systems and are likely to contribute significantly to the total pressure of the system (as well as playing an important role in star formation).

Absorption has been used to study molecular gas, and here we can study $\mathrm{H}_{2}$ directly through the many electronic transitions $(\sim 100)$ in the far ultraviolet region. Through this approach, molecular columns were determined in about 100 sight lines with Copernicus (Savage et al., 1977), and many more are being determined with the Far Ultraviolet Explorer (FUSE). The picture that has emerged for molecular gas is that it occupies an insignificant amount of the volume of the ISM, but it probably accounts for half of the mass.

One of the issues that must be addressed is the amount of molecular gas, which may be underestimated by a substantial amount. In the Milky Way, the Copernicus observatory showed that for $\mathrm{N}_{\mathrm{HI}}>5 \times 10^{20} \mathrm{~cm}^{-2}, \mathrm{H}_{2}$ is very prevalent and a significant fraction of $\mathrm{HI}$ ( $25 \%$ of the total $\mathrm{HI}+\mathrm{H}_{2}$ column; Savage et al., 1977). In a completely independent study, where molecular gas is identified through the 
absorption strength of the $\mathrm{HCO}^{+}$millimeter line, $\mathrm{HCO}^{+}$absorption is seen in 18/22 lines of sight below 39 degrees (Lucas and Liszt, 1996), which corresponds to column densities above $5 \times 10^{20} \mathrm{~cm}^{-2}$; this is consistent with the Copernicus result. In another independent study in yet another waveband, X-ray absorption toward extragalactic sight lines indicates substantial absorption when $\mathrm{N}_{\mathrm{HI}}>5 \times 10^{20} \mathrm{~cm}^{-2}$ (at latitudes below 40 degrees). The only possible ISM component that could be responsible for the extra absorption is $\mathrm{H}_{2}$, which would account for about $40 \%$ of the total neutral gas column (Arabadjis and Bregman, 1998), consistent with the above results.

These three results paint a consistent picture of the $\mathrm{H}_{2}$ content in the Solar circle, but it differs from the picture that one obtains from $\mathrm{CO}$ emission studies, which find that $\mathrm{CO}$ is not prevalent in the Solar neighborhood. Recently, there was a survey of $\mathrm{CO}$ at latitudes above 10 degreees (Hartmann, Magnani and Thaddeus, 1998), which detected little CO. They found a covering factor of 0.004-0.008 and using the usual $\mathrm{CO}$ to $\mathrm{H}_{2}$ conversion ratio, a mass surface density of $0.015-0.035 \mathrm{M}_{0}$ $\mathrm{pc}^{-2}$. The inferred $\mathrm{H}_{2}$ mass is about 50-130 times less than the $\mathrm{H}_{2}$ mass determined from the studies above.

The difference between these two determinations of the $\mathrm{H}_{2}$ mass can be understood bynoting that the interaction between molecules and the radiation field is different for $\mathrm{CO}$ and $\mathrm{H}_{2}$ at these column densities. This interaction is discussed by van Dishoek and Black (1988; and others), who show that for the ambient radiation field in the disk, $\mathrm{H}_{2}$ can shield itself against photodestruction when $\mathrm{A}_{\mathrm{V}}$ $>0.25$, which corresponds to $\mathrm{N}(\mathrm{HI})>5 \times 10^{20} \mathrm{~cm}^{-2}$. However, $\mathrm{CO}$ is still easily destroyed under these conditions, which leads to the situation where $\mathrm{CO}$ is nearly absent while $\mathrm{H}_{2}$ is still abundant. This can lead to values for the $\mathrm{CO}$ to $\mathrm{H}_{2}$ conversion factor $(\mathrm{X})$ that is orders of magnitude larger than the value derived for giant molecular clouds. Therefore, for the Galaxy, $\mathrm{CO}$ observations can lead to a significant underestimate of the molecular gas mass when using the typical value of X.

\section{Progress Toward a Self-Consistent Model of the ISM}

Our knowledge of the various interstellar components, although not perfect, is extensive and will allow us to build models and subsequently compare them to the data. The challenges to the modelers of the ISM are daunting both in scope and on the demands they place on codes and computers. To begin, the dynamic range of the density is enormous, from $10^{-4} \mathrm{~cm}^{-3}$ (hot gas) $-10^{4} \mathrm{~cm}^{-3}$ (molecular gas), an eight order of magnitude range. It might be possible to reduce this range a couple of order of magnitude, which is still enormous. There is a similar range in the temperature of the gas, although the range in the pressure is far less, only a couple orders of magnitude. In addition, magnetic fields must be included, as it is an integral part of the pressure term. Also, it can lead to heating through reconnection, and 
it is possibly of dynamic importance as well. Turbulence is essential in a realistic treatment, and if one is to model this, it must be carried out in three-dimensions, as this is required if turbulence is to evolve. All this occurs in a rotating galaxy where differential rotation can be important in the evolution of large structures such as superbubbles or large sheets of gas.

For the gas components, the heating and cooling processes determine the nature of the different components. Supernovae provide the dominant input of energy through shocks and possibly through thermal conduction between cold and hot gas. The radiative cooling of the gas is relatively easy to model, provided that regions are optically thin. However, heating of gas in the range $10-10^{4} \mathrm{~K}$ is driven by ultraviolet photon, through photoionization and photoelectron ejection off of grains. The transfer of UV photons will depend upon the absorbing properties of the gas and the ability of molecular gas to shield itself. In addition, molecules are formed and destroyed, and as this is one of the important connections with observations, astrochemistry must be dealt with.

To finish this list of relevant processes, there is a transfer of gas from the ISM to stars through star formation, and the ejection of gas from aging stars (the red giant phase) as well as those at the ends of their lives (planetary nebulae and supernovae).

This is a tremendously daunting list of processes that one must keep track of and the task is formidable, but there have been a wide range of approaches taken that have shed light on this problem. We will hear about many of these at this meeting so I will only briefly review a few of the successes and approaches. Parts of the problem can be broken off and studied fruitfully. For example, turbulence is most important on moderately scales small compared to the Galaxy and there has been a lot of activity in studying energy transfer. A different example is the flow of hot gas in galactic halos, where the scales are kpc, so one can ignore the detailed structure of cold gas as being unimportant to the main aspects of the flow.

There has been a good deal of progress understanding the role of turbulence in theprocess of energy transfer. This addresses the issue of how the mechanical energy of a magnetized fluid is transformed into thermal energy. Along these lines, an important theory is the Alfvén Wave Decay Model of Goldreich and Sridhar (1995) in which they study incompressible magnetized fluids. Previous 'traditional' theories posited the isotropy of the energy cascade in Fourier space, but as Goldreich and Sridhar point out, this cannot be true in the ISM because B fields are more easily bent on large than on small scales. Therefore, the energy cascade should be anisotripic in Fourier space, so in response to this, they developed a theory of strong turbulence of shear Alfvén wave. In their theory, there is a critically balanced cascade between linear wave periods and nonlinear turnover timescales, which results in a Kolmogorov-type spectrum. This has been further developed by these and other authors and numerical simulations support this picture (Cho, Lazarian and Vishniac, 2002; Lazarian, this meeting).

An important extension of these physical conditions occurs when the fluid becomes compressible. These conditions have been investigated numerically and 
although they find that the turbulence is also Kolmogorov-like, there are phenomena not possible in the incompressible case: shocks and density fluctuations. MacLow and coworkers (e.g., this meeting) suggest that the density fluctuations are important in that they are the regions that ultimately undergo gravitational collapse in the star formation process. In addition, they show that shocks can be of greater importance than Alfvén waves in transferring energy on these scales.

The large-scale numerical efforts to simulate that ISM have taught us a great deal and have the potential of tapping into the wealth of observational data available. These numerical simulations divide space into a large number of cells, each of which has a single value for the various fluid properties, such as density, temperature, velocities, and magnetic field strengths. These calculations are carried out on the fastest computers available and a limiting constraint is usually the number of cells used. If one wants to investigate a region large enough to contain superbubbles and halo gas (a scale of several $\mathrm{kpc}$ ), while still resolving relevant detail in the molecular component $(1 \mathrm{pc})$, the number of cells required becomes very great and the calculation timestep becomes unacceptably small; such calculations are unfeasible. This difficulty is now resolved by using larger and fewer cells in most of the calculation, but adding cells to regions that require a greater degree of resolution. This subgridding scheme is dynamic in that it number and location of the extra cells changes with time and location in the grid.

The results of these calculations in the past few years (e.g., de Avillez, 2000 and this meeting) show results that are common with older calculations (e.g., Rosen and Bregman, 1995), but with several new surprises. Gas is generally pushed into sheets of material with lower density gas separating the sheets. The sheets can be quite dense, especially where they intersect with other sheets and these are regions that are the denser $\mathrm{HI}$ clouds or $\mathrm{H}_{2}$. Supernovae tend to work collectively, creating large hot low density regions, which can be well-defined as superbubbles, or rather irregular regions. This hot gas will naturally rise, but it does not have a clear path out of the galaxy, since it interacts with (and becomes) the gaseous halo above the disk that is a consequence of the supernova heating.

For the Galactic value of the heat input from supernovae, the various scale heights for the different temperature components can be reproduced, but in addition to that, there are other important structural features that emerge. For example, these calculations produce hot superbubbles of approximately the size that is observed in the X-ray observations. Also, one finds the formation of chimneys in the HI component as well as the formation of HI 'mushrooms' that are observed (de Avillez, 2001a,b).

The simulations that focus on molecular clouds yield an appearance that, to visual inspection, is generically similar to CO maps found by observers. Also, these simulations appear to reproduce the observed size-linewidth relationship. These calculations necessarily include magnetic fields and turbulence (Ostriker, Stone and Gammie, 2001; Pavlovski et al., 2002) and there are significant morphological differences between the results of these investigations that we can identify whether 
or not these clouds lie in the strong or the weak turbulent limits. I am impressed with these calculations since I thought that numerical studies with this level of realism were still years away.

One of the long-standing disagreements in the development of a global model of the ISM has been the role of thermal conduction. Thermal conduction was introduced as a crucial component of these models by McKee and Ostriker (1977), probably the most often quoted paper on the subject. They argued that the undisturbed interstellar medium is naturally a two-density medium at nearly constant pressure: there are many dense cold HI clouds imbedded in a lower density warmer medium. A supernova shock races through the low density medium, heating it rapidly, but the shocks do not heat the dense clouds effectively, so there are dense clouds within a hot, high-pressure medium. The juxtaposition of hot and cold gas leads naturally to conductive heat transfer, provided that tangled magnetic fields do not reduce the effectiveness of the process. Heat conduction would lead to the transfer of the cold clouds to the hot medium. This process, in addition to providing an elegant mechanism by which one component is transformed into another, can act as a regulation mechanism. In addition, it seemed to solve an apparent problem with the size-mass relationship for SNRs, where they appeared to increase their mass more rapidly than the increase in their volume.

This theory seemed to solve many problems at once and was consistent with much of the data. However, the issue with the size-mass relationship for SNRs went away when Hamilton, Chevalier and Sarazin (1983) showed that the relationship could be reproduced if one included time-dependent ionization effects. In the discussion of the effectiveness of conduction, the central issue became the predicted 'intermediate temperature gas' that lies between the temperature of the hot gas and of the cold gas. Many of the important ions in this temperature range $\left(10^{5}-10^{7} \mathrm{~K}\right)$ have their resonance transitions at energies above $13.6 \mathrm{eV}$ and cannot be studied, but OVI, representative of gas near $10^{5.5} \mathrm{~K}$, is the most common heavy element and has strong transitions in the far ultraviolet region, a doublet centered at $1035 \AA$. While OVI absorption is detected, it is far weaker than predicted from the McKee-Ostriker picture (Shelton and Cox, 1994) and unless this deficit of OVI can be explained, the conflict with the theory is great enough to rule out the theory. We might add that if one were to replace thermal conduction with a mixing layer model (Slavin, Shull and Begelman, 1993), it would suffer the same problem since this process produces a significant amount of gas in the $10^{5}-10^{6} \mathrm{~K}$ range. This is good new for modelers in that it appears that they can ignore thermal conduction or mixing layers in their models.

Some of the other assumptions of the McKee-Ostriker model may not be present, such as the mixture of many cold clouds within a lower density medium. While there are undoubtedly small clouds of HI, such as the one that the Sun is located within (although it is not high density), observations and theory suggest that most of the cold gas will lie in sheets that are separated from the lower density gas, rather than being intertwined. 


\section{Confronting Theory with Observations}

The confrontation between observations and theory is at the center of the advancement in our knowledge. In the past, most of the comparisons have been with a few scalars that were extracted from the data, such as a density, temperature, pressure, or scale height. However, both observations and theory contain far more information that cannot adequately be summarized by a few scalars. For example, the Canadian Galactic Plane HI Survey has a vast amount of detailed structural information (and as a function of velocity), yet this richness is not being exploited in the comparison with models. Similar situations exist for molecular gas studies, where there are many excellent $\mathrm{CO}$ maps, and for the warm ionized component, where WHAM is producing beautiful $\mathrm{H} \alpha$ maps. This problem has faced other fields at some point in their history and there have been some interesting solutions to this problem. For example, in cosmology, a geometric method was developed (the 'genus' method; Gott, Melott and Kickinson, 1986) that could quantify the topology of the distribution of galaxies. The quantity that is fit in this case is a curve rather than a scalar, which is necessary to summarize the data. There have been a number of other methods as well but the most successful ones begin from physical principles and craft a statistical test around the most important physical quantities. An example of the opposite approach is to take some mathematical form that may or may not have physical significance, such as fractals, and perform an analysis based on it. In most cases, I have not learned much from such studies.

In an effort to progress toward these more complex analysis, Adams and Wiseman (1994) suggest an interesting approach that can be applied to molecular gas, but has broader applications. They discuss the analysis of maps (although their formalism is more general) whereby one would compare an observed map to a theoretical one. The difference between two maps is measured as a distance in map space, with the goal of minimizing this distance. They give an example of this method applied to IRAS maps of molecular clouds in star-forming regions (Wiseman and Adams, 1994). Whereas there are other possible approaches even to this problem (e.g., a 'tree' algorithm for molecular cloud maps; Houlahan and Scalo, 1992), it points out that there are more sophisticated techniques that need to be employed if we are to compare the new sets of theoretical models to the wonderfully detailed maps of data.

\section{References}

Adams, F.C. and Wiseman, J.J.: 1994, ApJ 435, 693.

Arabadjis, J.S. and Bregman, J.N.: 1998, ApJ 510, 806.

de Avillez, M.A.: 2000, MNRAS 315, 479.

de Avillez, M.A. and Berry, D.L.: 2001, MNRAS 328, 708.

de Avillez, M.A. and MacLow, M.-M.: 2001, ApJ 551, L57.

Blitz, L., Spergel, D.N., Teuben, P.J., Hartmann, D. and Burton, W.B.: 1999, ApJ 514, 818. 
Bregman, J.N., Kelson, D.D. and Ashe, G.A.: 1992, ApJ 409, 682.

Cho, J., Lazarian, A. and Vishniac, E.T.: 2002, ApJ 564, 291.

Cox, D.P.: 1981, ApJ 245, 534.

Van Dishoeck, E.F. and Black, J.H.: 1988, ApJ 334, 771.

Goldreich, P. and Sridhar, S.: 1995, ApJ 438, 763.

Gott, III, J.R., Melott, A.L. and Kickinson, M.: 1986, ApJ 306, 341.

Hamilton, A.J.S., Chevalier, R.A. and Sarazin, C.L.: 1983, ApJS 51, 115.

Hartmann, D., Magnani, L. and Thaddeus, P.: 1998, ApJ 492, 205.

Houlahan, P. and Scalo, J.M.: 1992, ApJ 393, 172.

Kulkarni, S.R. and Heiles, C.: 1988, in: G.L. Verschuur and K.I. Kellermann (eds.), Galactic and Extragalactic Radio Astronomy, 2nd edn.; Springer, Berlin, p. 95.

Lucas, R. and Liszt, H.: 1996, A\&A 307, 237.

McKee, C.F. and Ostriker, J.P.: 1977, ApJ 218, 148.

Ostriker, E.C., Stone, J.M. and Gammie, C.F.: 2001, ApJ 546, 980.

Pavlovski, G., Smith, M.D., MacLow, M-M. and Rosen, A.: 2002, MNRAS 337, 477.

Reynolds, R.J.: 1989, ApJ 339, L29.

Reynolds, R.J.: 1991, ApJ 372, L17.

Reynolds, R., Tufte, S.L., Haffner, L.M., Jaehnig, K. and Percival, J.W.: 1998, Pub. Astron. Soc. Aust. $15,14$.

Rosen, A. and Bregman, J.N.: 1995, ApJ 440, 634.

Sanders, W.T. et al.: 2002, AAS, 201.4705.

Savage, B.D., Bohlin, R.C., Drake, J.F. and Budich, W.: 1977, ApJ 216, 291.

Savage, B.D. et al.: 2003, ApJS, in press.

Sembach, K.R.: 2003, ApJS, in press.

Shelton, R.L. et al.: 2001, ApJ 560, 730.

Shelton, R.L.: 2002, ApJ 569, 758.

Shelton, R.L. and Cox, D.P.: 1994, ApJ 434, 599.

Slavin, J.D., Shull, J.M. and Begelman, M.C.: 1993, ApJ 407, 83.

Snowden, S.L., Egger, R., Finkbeiner, D.P., Freyberg, M.J. and Plucinsky, P.P.: 1998, ApJ 493, 715.

Taylor, J.H. and Cordes, J.M.: 1993, ApJ 411, 674.

Tufte, S.L., Wilson, J.D., Madsen, G.J., Haffner, L.M. and Reynolds, R.J.: 2002, ApJ 572, L153.

Wakker, B.P. et al.: 2003, ApJS, in press.

Wakker, B.P., Oosterloo, T.A. and Putman, M.E.: 2002, AJ 123, 1953.

Weiner, B.J., Vogel, S.N. and Williams, T.B.: 2002, in: J.S. Mulchaey and J. Stocke (eds.), Extragalactic Gas at Low Redshift, ASP Conference Proceedings Vol. 254, ASP, San Francisco, p. 256. 